Check for updates

Cite this: Phys. Chem. Chem. Phys., 2017, 19, 16124

Received 28th March 2017

Accepted 5th June 2017

DOI: $10.1039 / c 7 c p 01994 g$

rsc.li/pccp

\title{
Interfacial and volumetric sensitivity of the dry sintering process of polymer colloidal crystals: a thermal transport and photonic bandgap study $\dagger$
}

\begin{abstract}
Fabian A. Nutz and Markus Retsch (D) *
We introduce the in situ characterization of the dry sintering process of face-centred cubic colloidal crystals by two complementary techniques: thermal transport and photonic stopband characterization. Therefore, we employed time-dependent, isothermal laser flash analysis and specular reflectivity experiments close to the glass transition temperature of the colloidal crystal. Both methods yield distinctly different time constants of the film formation process. This discrepancy can be attributed to a volume- (photonic stopband) and interface-driven (thermal transport) sensitivity of the respective characterization method. Nevertheless, both methods yield comparable apparent activation energies. Finally, we extended the sintering process characterization to further polymer compositions, with vastly different glass transition temperatures. We could show that the film formation rate is governed by the viscoelastic properties of the polymers at the respective annealing temperature.
\end{abstract}

\section{Introduction}

Colloidal crystals are an intensely studied material class in the focus of a wide variety of recent research. ${ }^{1-6}$ They are employed in many active research fields such as photonics and phononics, ${ }^{7-9}$ lithography, ${ }^{10}$ defined particle-immobilization ${ }^{11}$ and many more. ${ }^{12}$ Recently, colloidal crystals have also drawn attention in the field of thermal transport in nanostructured materials. ${ }^{13,14}$ Due to their highly-defined structure on the colloidal length scale, they can serve as a model system to get a deeper understanding of thermal transport in porous, particulate matter. Heat flow through a colloidal crystal is extremely sensitive to the size and bonding strength of the interfaces between neighbouring particles. This characteristic can be utilized to trace particle dry sintering within polymer colloidal crystals at temperatures above the glass transition $T_{\mathrm{g}}$ of the polymer.

Usually, polymer colloidal crystals are formed of closed packed arrays from monodisperse polymer particles with a diameter ranging from 100-1000 $\mathrm{nm}$. These crystals are predominantly assembled from aqueous particle dispersions. Based on the widespread application of particle dispersions as paints, paper

University of Bayreuth, Physical Chemistry - Polymer Systems, Universitaetsstr. 30, 95447 Bayreuth, Germany. E-mail: Markus.Retsch@uni-bayreuth.de

$\dagger$ Electronic supplementary information (ESI) available: Scheme of stages of film formation; scheme and description of laser flash analysis experiment; timedependent behaviour of the thickness at different annealing times; SEM sideviews of annealed colloidal films; additional time-dependent UV-vis and XFA experiments, rheology strain sweeps, temperature-dependent storage and loss modulus. See DOI: 10.1039/c7cp01994g coatings and adhesives, a deep understanding of film formation and particle sintering is necessary, to achieve the desired performance. ${ }^{15-17}$ The film formation process of latex dispersions can be generally separated into three stages (see Fig. S1, ESI $\dagger$ ): ${ }^{16}$ in stage I the particles self-assemble during the evaporation of water. If the particles possess a sufficient narrow size distribution, they assemble into a colloidal crystal. stage II only occurs above the minimum film-forming temperature (MFT). The MFT is strongly related to the glass transition temperature $T_{\mathrm{g}}$ of the polymer. Exceeding $T_{\mathrm{g}}$ leads to the softening of the particles, allowing them to deform into dodecahedrons, where each facet is in full contact to a facet of the neighbouring particles. This results in optically clear polymer films due to the loss of the refractive index contrast within the structure. For most applications, e.g. paints, the MFT is below room temperature, resulting in wet sintering of the particles as the water evaporates. If the particles possess a $T_{\mathrm{g}}$ above room temperature, the polymer is still in its glassy state and deformation of the particles is prevented during water evaporation. Heating the assembly above its $T_{\mathrm{g}}$ leads to dry sintering of the particles. ${ }^{18-21}$ The closure of the porosity and the formation of mutual full contact areas is not only driven by the viscosity of the polymer at the specific temperature, but also by the surface tension between the water-polymer or the air-polymer interface in the case of wet and dry particle sintering, respectively. ${ }^{19,22,23}$ Thus, the particle deformation in stage II is additionally size-dependent. ${ }^{24}$ In stage III, polymer diffusion across the particle-particle interfaces takes place, yielding a continuous polymer film.

During the past decades, a range of methods, such as smallangle neutron scattering (SANS), ${ }^{25-31}$ direct non-radiative energy 

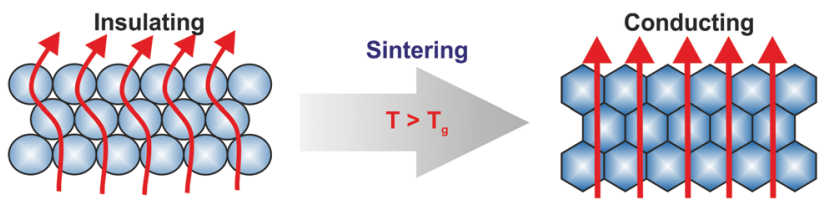

Scheme 1 Schematic illustration of the increasing thermal conductivity during sintering of a polymer colloidal crystal. Due to the small contact points, the crystal is thermally insulating at temperatures below its $T_{\mathrm{g}}$. By exceeding $T_{\mathrm{g}}$ the particles deform into dodecahedrons with every facet in full contact to the facet of the neighbouring particle. This leads to an increased thermal transport through the structure.

transfer (DET), ${ }^{32-36}$ small-angle X-ray scattering (SAXS), ${ }^{21,37-40}$ atomic force microscopy (AFM) $)^{19,41,42}$ and solid state $\mathrm{NMR}^{42,43}$ haven been established to investigate latex film formation and dry particle sintering. Most of these methods need a quite elaborate experimental setup or a specific labelling with dyes/ deuterated compounds of the sample. Some of these methods are furthermore restricted to assemblies comprising a sufficient particle size.

Hence, in this work, we want to contribute to the understanding of the dry sintering process by a novel approach. Therefore, we monitor changes of the thermal transport properties of polymer colloidal crystals by time-dependent laser flash analysis (LFA) for the first time. This method is capable to follow the sintering process in situ. It can be applied to particles of any size and no labelling of the polymers or the use of deuterated monomers is needed. Our system is based on monodisperse nanoparticles comprising a random copolymer of methyl methacrylate (MMA) and $n$-butyl acrylate ( $n \mathrm{BA})$. As we demonstrated in a recent publication on polystyrene colloidal crystals, the thermal transport in such structures is strongly governed by the small contact points between the particles. ${ }^{13}$ These interfaces serve as geometrical constrictions for heat to travel through the material. Therefore, the size of these contact points is crucial for the thermal transport. Beyond the glass transition of the polymer, the polymer chains become mobile. This leads to a strong enlargement of the contact points between the adjacent spheres and thus, to a drastically increase of $\sim 300 \%$ of the thermal conductivity (Scheme 1 ).

We take advantage of this effect and trace the dry sintering of $20 \mathrm{vol} \%-n$ BA-co-80 vol\% MMA colloidal crystals $\left(T_{\mathrm{g}} \sim 74{ }^{\circ} \mathrm{C}\right)$ at 70,75 and $80{ }^{\circ} \mathrm{C}$. The high ordering within the crystals additionally allows following the sintering process by time-dependent UV-vis reflectivity measurements, recording the decrease of the Bragg reflection. Whereas LFA is extremely sensitive to changes at the interfaces of the crystal, UV-vis is mainly responding to volume changes, influencing the refractive index contrast. We finally show the sintering process to be independent of the polymer composition, but being mainly governed by the viscoelastic properties of the respective polymer.

\section{Materials and methods}

\section{Materials}

Methyl methacrylate (MMA, 99\%, Aldrich) and $n$-butyl acrylate $(n \mathrm{BA} ; \geq 99 \%$, Aldrich) were purified by filtration over an alumina column (activated, basic, Brockmann I, Sigma-Aldrich) prior to the synthesis. Potassium peroxodisulfate (KPS, $\leq 99 \%$, Sigma-Aldrich) and 4-styrenesulfonic acid sodium salt hydrate (NaSS, 99\%, Aldrich) were used as received. Throughout the synthesis and purification steps, water was taken from a Millipor Direct Q3UV unit.

Synthesis. Copolymer particles were synthesized by emulsifierfree emulsion polymerization according to literature. ${ }^{44}$ In a typical synthesis, $240 \mathrm{~mL}$ milliQ water and $10 \mathrm{~mL}$ of total monomer mixture (8 mL MMA and $2 \mathrm{~mL} n$-BA for $20 \mathrm{vol} \% n \mathrm{BA}-\mathrm{co}-80 \mathrm{vol} \%$ MMA particles) were charged in a $250 \mathrm{~mL}$ 3-neck flask, equipped with a reflux condenser and a gas inlet. The mixture was heated to $80{ }^{\circ} \mathrm{C}$ and stirred under a constant argon flow. The mixture was allowed to equilibrate for $15 \mathrm{~min} .10 \mathrm{mg}$ of NaSS and $200 \mathrm{mg}$ of KPS were separately dissolved in $5 \mathrm{~mL}$ milliQ water each. After equilibration, the aqueous solution of NaSS was added, and the mixture was allowed to further equilibrate for $5 \mathrm{~min}$. The polymerization was started by rapidly adding the KPS solution to the mixture. The reaction was carried out overnight at $80{ }^{\circ} \mathrm{C}$ under argon atmosphere. To remove residual educts ( $n \mathrm{BA}, \mathrm{MMA}$, NaSS, KPS), the particles were dialyzed against milliQ water for three days, changing water twice a day. For the synthesized polymers, the molecular weight can be expected to be in the range of 90 to $120 \mathrm{~kg} \mathrm{~mol}^{-1} \cdot{ }^{45}$ Calculation of the theoretical glass transition temperature were done by the Fox equation assuming a $100 \%$ conversion of the monomers.

Crystal assembly. Colloidal crystals were fabricated by slowly drying a given amount of particle dispersions in a PTFE beaker for several days under ambient conditions. Disk-shaped colloidal crystals with a diameter of $\sim 20 \mathrm{~mm}$ were obtained after drying (Fig. 1A).

\section{Methods}

Dynamic light scattering. The hydrodynamic diameter of the particles and their size distribution were determined by dynamic light scattering (DLS) on dilute aqueous particle dispersions. Measurements were performed on a Malvern Zetasizer with $175^{\circ}$ backscattering geometry.

Light microscopy. Bright field light microscopy images onto the edges of split colloidal crystals were recorded on a Carl Zeiss Axio Imager.A2m light microscope equipped with an AxioCam Icc 1 camera.

Scanning electron microscopy. Scanning electron microscopy (SEM) was performed on a Zeiss Leo 1530 electron microscope to determine the hard sphere diameter of the particles. Dilute particle dispersions were drop-casted on a silicon wafer and sputtered with $1.3 \mathrm{~nm}$ platinum. Additionally, side-view SEM images along the edges of split colloidal crystals were recorded to investigate the order of the particles within the interior of the monoliths.

Differential scanning calorimetry. Heat capacity determination was carried out on a TA Instruments Q1000 differential scanning calorimeter according to ASTM E1269. Three individual measurements were performed under a nitrogen flow of $50 \mathrm{~mL} \mathrm{~min}{ }^{-1}$ at a heating rate of $20 \mathrm{~K} \mathrm{~min}^{-1}$. Two heating cycles were conducted between $-40{ }^{\circ} \mathrm{C}$ and $200{ }^{\circ} \mathrm{C}$. The specific heat capacity was evaluated from the second heating cycle. Based on the low 

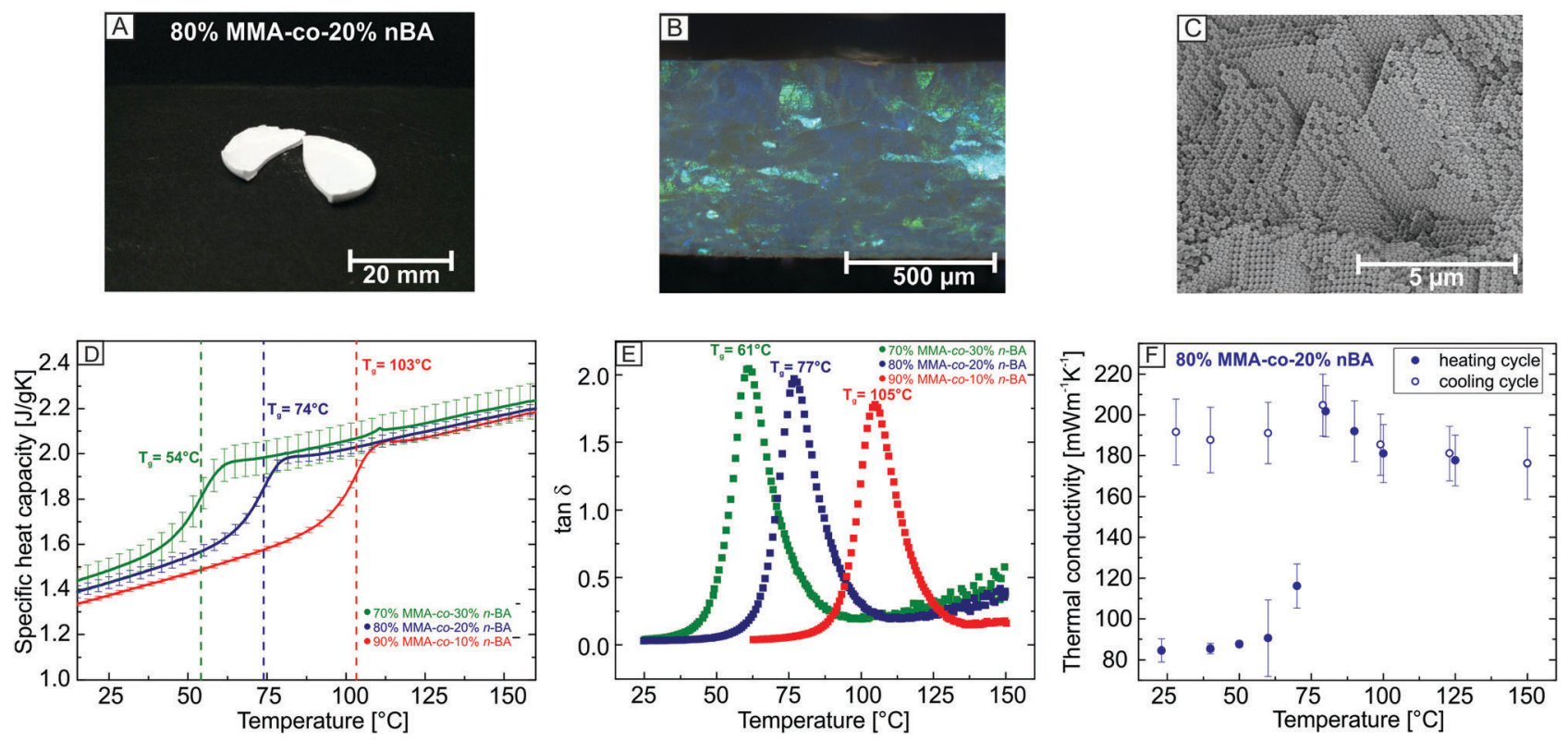

Fig. 1 (A) Photograph of a free-standing, self-assembled colloidal crystal monolith. (B) Side-view optical microscopy image along the edge of a split colloidal crystal. The opalescence is indicative of a high crystalline order. (C) Side-view SEM image of the interior of the colloidal crystal monolith confirming the long range, crystalline order. (D) Temperature-dependent heat capacity and $T_{\mathrm{g}}$ of three copolymers with increasing $n B A$ content. (E) $T_{\mathrm{g}}$ analysis based on the mechanical properties of the bulk polymer. (F) Temperature-dependent thermal conductivity of the colloidal crystal. By exceeding $T_{\mathrm{g}}$ of the polymer a drastic increase of the thermal conductivity is visible.

weight content of the gas phase within the crystal, only the heat capacity of the polymer is considered in the calculation of the thermal conductivity.

Density determination. The density of the pristine colloidal crystal was determined from the mass and volume of the monoliths. The mass was determined by weighting the crystals. The volume was measured on a Keyence V-3100 3D digital macroscope. The density of the molten crystals has been obtained by a buoyancy balance according to Archimedes principle.

Rheology. The storage and loss modulus of the polymers were determined using oscillating rotational rheology with plate-to-plate geometry at a frequency of $1 \mathrm{rad} \mathrm{s}^{-1}$ in a temperature range of 25 to $150{ }^{\circ} \mathrm{C}$. Measurements were performed on diskshaped samples with a diameter of $8 \mathrm{~mm}$. Strain sweeps were performed before the measurements to determine the linear viscoelastic regime of the samples (Fig. S5a, ESI $\dagger$ ). The samples were prepared by hot-pressing dry nanoparticle powder at $150{ }^{\circ} \mathrm{C}$ in a vacuum hot-press, to ensure no enclosure of air bubbles within the samples.

UV-VIS. Temperature-dependent UV-vis reflectivity measurements were conducted on an Agilent Cary 5000 with Universal Measurement Accessory equipped with a heat stage. UV-vis experiments were carried out on colloidal crystals, dip-coated from a $3 \mathrm{wt} \%$ particle dispersion on $170 \mu \mathrm{m}$ glass slides. Reflectivity spectra were recorded from $350-550 \mathrm{~nm}$ at an angle of $10^{\circ}$ between sample and incident light beam. The range of the detected wavelength is chosen as such to detect Bragg reflection of the colloidal crystals.

Laser flash analysis. Laser flash analysis was performed on a Linseis XFA 500 XenonFlash apparatus equipped with an InSb infrared detector to obtain the thermal diffusivity $\alpha$ of the colloidal crystals. Previous to the measurement the sample surfaces were coated with a 100-200 nm metal layer. The metal layer serves as an optical blocking layer and prevents light transmission through the colloidal crystal. Additionally, the samples were coated with a thin graphite layer $(<15 \mu \mathrm{m})$ to ensure a good absorbance at the bottom, and a high emissivity at the top side of the sample. Due to the small thickness of the coating, compared to the sample thickness $(\sim 800 \mu \mathrm{m})$ the influence of the coating on the measurements is negligible. Measurements were performed in helium atmosphere at a pressure of 950 mbar to ensure a homogenous temperature distribution inside the sample chamber. The raw data was fitted with the radiation fit model provided by the software Aprosoft Laser Flash Evaluation v.1.06. Measurements were performed on at least three samples.

Thickness determination. The thicknesses of the colloidal crystals were determined on a Mitutoyo Litematic VL-50 equipped with a heat stage. The sample thickness was measured before and after each laser flash experiment. For time-dependent thickness determination, a home-built heat stage was used to provide a comparable thermal environment to the laser flash measurements.

\section{Results and discussion}

Copolymer particles with a diameter of $214 \pm 7 \mathrm{~nm}$ determined by SEM were synthesized by emulsifier-free emulsion polymerization. Colloidal crystals of these particles were self-assembled by drying the dispersion at ambient conditions. Typically, disc-shaped, freestanding colloidal crystals with a thickness of several hundred 
micrometres and a diameter of $\sim 20 \mathrm{~mm}$ were obtained with this method (Fig. 1A).

Optical microscopy side-view images of typically obtained monoliths are shown in Fig. 1B. A strong opalescence is visible throughout the entire monolith. This demonstrates the high order of the particles within the crystal on a length scale of several hundred micrometres. SEM confirms the high degree of crystallinity of the crystal (Fig. 1C). From optical microscopy and SEM, the samples under investigation can be regarded as fully crystalline. The polymer microstructure, however, is of course in a completely amorphous state due to the atactic polyacrylate backbone.

The temperature-dependent heat capacity of three different $n$ BA-co-MMA copolymers is displayed in Fig. 1D. A glass transition temperature of $\sim 54{ }^{\circ} \mathrm{C}, \sim 74{ }^{\circ} \mathrm{C}$ and $\sim 103{ }^{\circ} \mathrm{C}$ can be determined (Fig. 1D, dashed line). This is in almost perfect agreement with the prediction of $53{ }^{\circ} \mathrm{C}, 74{ }^{\circ} \mathrm{C}$ and $99{ }^{\circ} \mathrm{C}$, calculated by the Fox equation. The glass transition temperature systematically decreases with increasing $n \mathrm{BA}$ content due to a higher rubber content within the final copolymer. The $T_{\mathrm{g}}$ was further confirmed by the temperature-dependent mechanical properties of bulk copolymer films (Fig. 1E). The peak of $\tan \delta$ gave values of $61{ }^{\circ} \mathrm{C}, 77^{\circ} \mathrm{C}$, and $105{ }^{\circ} \mathrm{C}$, respectively.

The thermal conductivity of monoliths from $20 \mathrm{vol} \% n \mathrm{BA}$ copolymer particles was measured by laser flash analysis. A schematic setup of this method and details about the measurement can be found in Fig. S2 (ESI $\dagger$ ). A typical measurement to obtain the thermal diffusivity is finished after roughly 10 seconds, depending on the thickness of the crystals. The temperature-dependent, mean thermal conductivity of three individual samples is presented in
Fig. 1F. 20 vol\% $n$ BA-co-80 vol\% MMA colloidal crystals show a low thermal conductivity of $84 \pm 2 \mathrm{~mW} \mathrm{~m}^{-1} \mathrm{~K}^{-1}$ at $25{ }^{\circ} \mathrm{C}$. By exceeding the $T_{\mathrm{g}}$ of the constituting polymer, the mobility of the polymer chains strongly increases, resulting in a fast sintering of the particles. Consequently, the thermal conductivity of the crystal increases in a step-like, irreversible fashion due to a strong enlargement of the particle-particle interfaces.

To investigate the dry sintering process, this transition is monitored by isothermal, time-resolved LFA and UV-VIS reflectivity measurements at temperatures close to the glass transition temperature. The dry sintering was followed at 70, 75 and $80{ }^{\circ} \mathrm{C}$. For time-dependent laser flash analysis, the changes in sample thickness have to be assessed separately (for details see ESI, $\dagger$ Fig. S3a). The time-dependent behaviour of a laser flash raw signal and the intensity of the Bragg peak at $75{ }^{\circ} \mathrm{C}$ are displayed in Fig. 2A and B (additional raw data is provided in Fig. S4, ESI $\dagger$ ).

For both methods, a qualitatively comparable behaviour could already be deduced by the time-dependent laser flash raw signal and the reflectivity spectra. The laser flash raw signal (Fig. 2A) increases in signal intensity with elapsing time. This is based on a decrease of crystal thickness due to the loss of porosity during film formation. Moreover, the signal maximum is shifted to shorter times. This time shift arises from an increased thermal transport through the sample due to the enlargement of the particle-particle interfaces. This leads to smaller $t_{1 / 2}$ values and thus, to an increase of $\alpha$ with elapsing time.

An intense Bragg peak of the neat colloidal crystal with a maximum at a wavelength of $473 \mathrm{~nm}$ is visible at the beginning of the experiment (Fig. 2B). This maximum corresponds to a
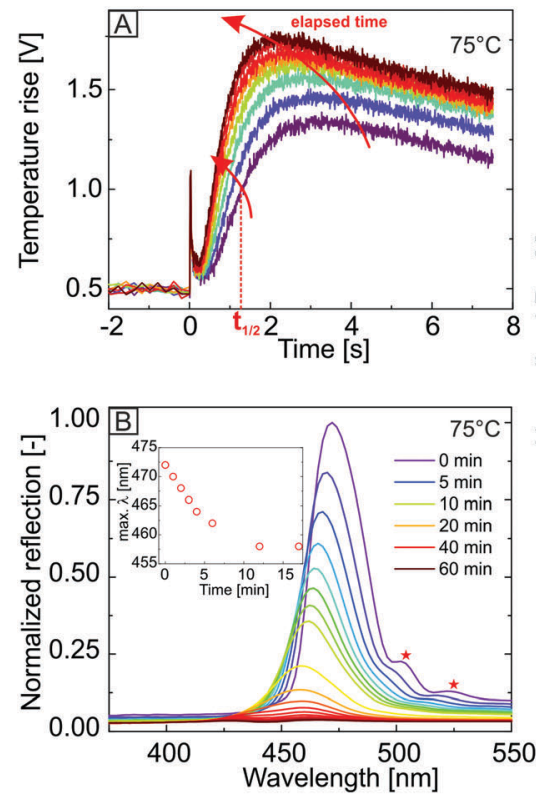
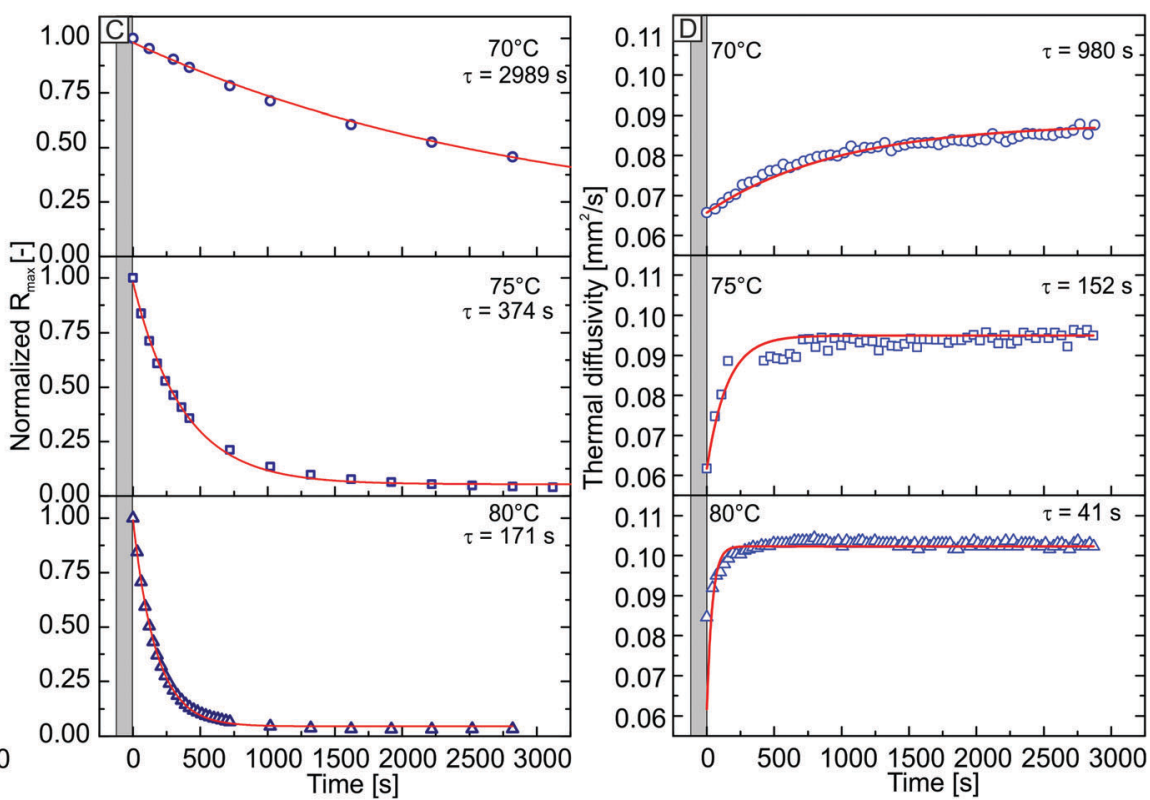

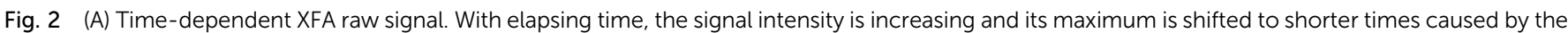

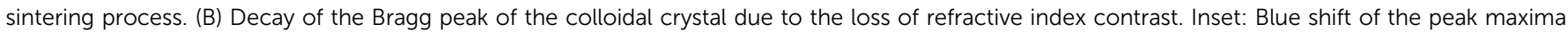

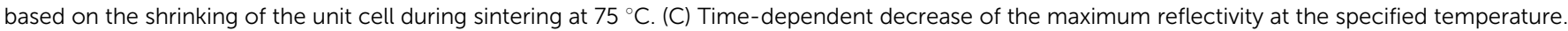

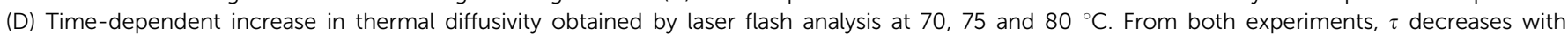
increasing temperature. 
colloidal crystal formed by particles with a diameter of $213 \mathrm{~nm}$ calculated by Bragg's law, assuming an fcc-symmetry and an effective refractive index of 1.379 for the structure. This is in very good agreement with the experimental particle diameter of $214 \pm 7 \mathrm{~nm}$ determined by SEM. In addition, the spectra exhibit Fabry-Pérot fringes (Fig. 2B, red stars) caused by interference of light waves, reflected at the crystal/air and crystal/substrate interface. This indicates a high order and homogeneity of the sample. With increasing annealing time, the intensity of the Bragg peak decreases. This is caused by the onset of polymer flow into the voids of the crystal. Due to the resulting loss of porosity, the refractive index contrast vanishes, leading to a decreasing Bragg peak intensity. Additionally, a blue shift of the peak's maxima is visible in the spectra. The evaluation of blue shift of the peak maxima for UV-vis experiments at $75{ }^{\circ} \mathrm{C}$ is displayed in the inset for Fig. 2B, for the first $1000 \mathrm{~s}$. At longer sintering times, the allocation of the peak maxima was not possible due to peak broadening. The blue shift can be ascribed to the deformation of the polymer particles into dodecahedrons during the sintering, leading to an isotropic shrinking of the crystal's unit cell. Therefore, the lattice parameter of the crystal decreases, indicating a blue shift of the spectra.

The time-dependent decay of the maximum intensity of the Bragg peak at 70, 75 and $80{ }^{\circ} \mathrm{C}$ are shown Fig. 2C. The grey area marks the time needed for thermal equilibration. The isothermal condition was typically reached within two minutes. We found the decrease of the Bragg peak intensity to follow a singleexponential behaviour (Fig. 2C, red lines). The fit function yields a time constant $\tau$, which is utilized to describe the rate of the film formation process at different temperatures. With increasing annealing temperature, the decay rate of the Bragg peak increases. This results in a decrease of the extracted time constants. Noteworthy, the Bragg peak reflectivity of the colloidal crystal vanishes almost completely after $\sim 50$ minutes for annealing temperatures of $75{ }^{\circ} \mathrm{C}$ and $80{ }^{\circ} \mathrm{C}$, whereas a strong reflection remains within the spectra for measurements at $70{ }^{\circ} \mathrm{C}$ (Fig. 2C). This is based on a preserved crystalline structure within the sample, as shown by SEM side-view micrographs in Fig. S3b (ESI $\dagger$ ) of the annealed colloidal crystal. For crystals sintered at $70{ }^{\circ} \mathrm{C}$, it is still possible to deduce the crystalline structure as well as the original, spherical shape of the particles. For samples annealed at $75{ }^{\circ} \mathrm{C}$ and $80{ }^{\circ} \mathrm{C}$, one is able to retrace the lattice planes but the interstitial voids between the particles are completely filled with polymer.

The thickness-corrected, time-dependent thermal diffusivity measured at 70, 75 and $80{ }^{\circ} \mathrm{C}$, is displayed in Fig. 2D. Within the equilibration period (grey area), it was not possible to perform any measurement. For every time-dependent isothermal series, the thermal diffusivity $\alpha$ of the colloidal crystals exhibit a comparable behaviour. Whereas $\alpha$ strongly increases in the first minutes of the experiment, it plateaus after a certain period. The obtained thermal diffusivity consequently follows a singleexponential behaviour for each isothermal condition (Fig. 2D, red line). Analogously to the UV-vis experiment, $\tau$ decreases with increasing temperature. This can be ascribed to a higher mobility of the polymer chains at higher temperatures, which results in a faster enlargement of the interparticle contact points and therefore, to a faster increase of the thermal transport through the structure.

The time constants $\tau$ from the UV-vis measurement are significantly higher than the values received from XFA, indicating a slower sintering process, observed by the UV-vis experiments. We attribute this predominantly to the following reason: the thermal transport properties of the colloidal crystal are mainly driven by the interfaces between the polymer particles. Even small changes at these interfaces by a slight interfacial fusion of the polymer can already lead to a strongly increased thermal diffusivity through the structure. On the contrary, the film formation process monitored by UV-vis is driven by changes of the volume affecting the porosity of the structure. The polymer has to flow into the voids of the crystal structure to induce a change of the refractive index contrast. In this case, the polymer needs to creep over longer distances and a larger amount of material needs to start moving in order to affect the crystal's reflectivity. It is further important to note that, despite the macroscopic dimensions of the colloidal crystal film, the sintering process happens homogeneously throughout the bulk of the colloidal crystal.

We can use the temperature-dependent rate constants $\tau$ to determine an apparent activation energy $E_{\mathrm{a}}$ for the relaxation of the polymer. The Arrhenius plot based on the extracted $1 / \tau$ values of both, UV-vis and XFA experiments is displayed in Fig. 3A. The values from XFA are systematically located at higher values than the data received from UV-vis due to the higher interfacial sintering rate. Both data sets show a linear behaviour. From the slope of the linear fit (Fig. 3A, red lines), $E_{\text {a }}$ can be estimated according to the Arrhenius equation. We found $E_{\mathrm{a}}$ from UV-vis and XFA to be comparable within the experimental error with $268 \mathrm{~kJ} \mathrm{~mol}^{-1} \pm 52 \mathrm{~kJ} \mathrm{~mol}^{-1}$ and $283 \mathrm{~kJ} \mathrm{~mol}^{-1} \pm 9 \mathrm{~kJ} \mathrm{~mol}^{-1}$, respectively. The much higher error from the UV-vis analysis originates from the few data points used for fitting. Therefore, both methods yield a comparable, apparent activation energy for the sintering process, but constitute distinctly different pathways. The interfacial fusion happens about three time faster than the bulk void filling.

The received $E_{\mathrm{a}}$ values are comparably high. This can be ascribed to several reasons. First, the relaxation of the polymer at temperatures near $T_{\mathrm{g}}$ can be understood as a beginning cooperative intermolecular motion. Since the measurements were performed only slightly above $T_{\mathrm{g}}$, this process might consume high energies due to the beginning dis-entanglement of the nearly glassy polymer chains. Second, the temperature range used for the linear fitting is quite limited, which can lead to an only apparent linear behaviour of the data. Third, since the measurements were performed on an amorphous polymer close to $T_{\mathrm{g}}$, the viscosity might rather follow a Williams-Landel-Ferry behaviour than an Arrhenius behaviour.

In a further step, we correlated the results obtained by XFA with the viscoelastic properties of three other $n \mathrm{BA}-c o$-MMA colloidal crystals possessing different polymer compositions. By varying the $n \mathrm{BA}$ content between 10 to $30 \mathrm{vol} \%$ the glass transition can be shifted to lower temperatures, according to the Fox equation. The sintering process should be independent 

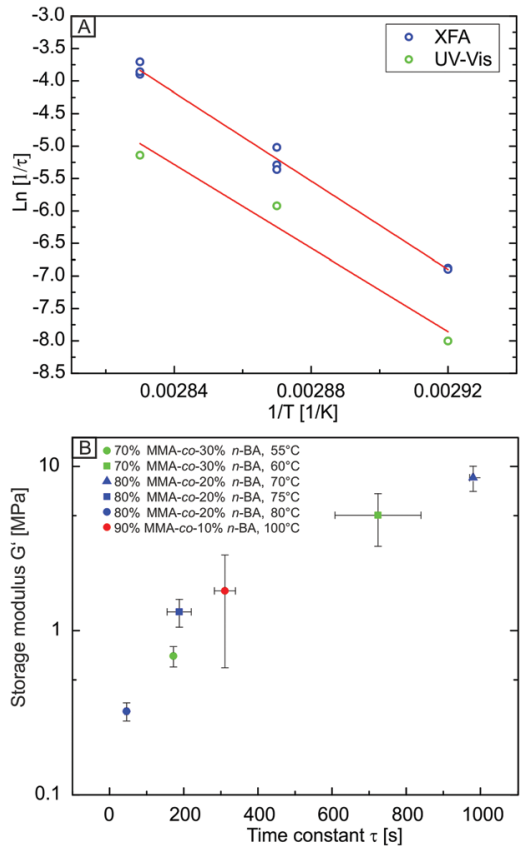

Fig. 3 (A) Arrhenius plot based on the fitted $\tau$ values from timedependent UV-vis (green) and XFA (blue) experiments. Whereas the absolute $1 / \tau$ values differ between the XFA and UV-vis experiment, the respective slopes are comparable. (B) Correlation between the storage modulus and time constants $\tau$ obtained from laser flash analysis. $\tau$ strongly depends on the viscoelastic properties of the polymer rather than its composition

of the actual polymer composition, but it certainly depends on the viscosity of the polymer at a given temperature. Moreover, it depends on the surface tension between the air-polymer interface $^{22,23}$ and consequently on the particle size. ${ }^{24}$ The differently composed particles were synthesized such that they all possess an almost equal diameter ranging between $182 \mathrm{~nm}$ and $215 \mathrm{~nm}$. Thus, the influence of the surface tension and size is nearly negligible for the comparison of the sintering process of the differently composed colloidal crystals. Furthermore, based on the emulsion polymerization process used, one can also expect comparably high molecular weights among all polymer compositions. $^{45}$

The temperature-dependent storage and loss moduli of the investigated polymers are shown in Fig. S5b (ESI $\dagger$ ). All copolymers under investigation show a similar $G^{\prime}$ and $G^{\prime \prime}$ temperature dependence, which is typical for amorphous, thermoplastic polymers. Depending on the $n \mathrm{BA}$ content of the polymer, the moduli are shifted to lower temperatures. This is in accordance with a decreasing glass transition temperature with increasing $n$ BA content $\left(103{ }^{\circ} \mathrm{C}, 74{ }^{\circ} \mathrm{C}\right.$, and $54{ }^{\circ} \mathrm{C}$ for 10,20 , and $30 \mathrm{vol} \%$ $n \mathrm{BA}$, respectively). We then correlate the obtained time constants $\tau$ with the temperature-dependent storage modulus $G^{\prime}$ of the polymer (Fig. 3B). A master-curve is obtained for all polymer compositions, where $\tau$ only depends on the storage modulus in a monotonic, but non-linear way. Thus, the temperaturedependent viscoelastic properties of the constituting polymer can be used to estimate the time needed for the interface- and volume-driven sintering process.

\section{Conclusion}

We investigated the dry sintering process of well-ordered colloidal crystals via two label-free and size-independent methods. Using UV-vis spectroscopy to monitor changes in the refractive index environment and laser flash analysis to monitor changes of the thermal diffusivity through such a colloidal ensemble, we were able to follow the film formation in real time. We find a comparable film formation activation barrier for both cases of about $270 \mathrm{~kJ} \mathrm{~mol}^{-1}$. The characteristic film formation rate can be described by a time constant $\tau$. This time constant decreases with increasing temperature relative to the $T_{\mathrm{g}}$ of the polymer under investigation. The time constants obtained from the UV-vis experiment is systematically higher compared to the XFA analysis, which we attribute to the difference between an interface- and a volume-driven response. Finally, using three different polymer compositions, we were able to show that the film formation rate is only a function of the viscoelastic properties of the polymers forming the particles. We are convinced that the analysis of thermal transport properties will be used in the future for the characterization of other particulate systems, where not only kinetic but also structural information is necessary.

\section{Acknowledgements}

The authors thank Ute Kuhn for help with DSC and rheological experiments. This project was funded by the Volkswagen Foundation (Lichtenberg professorship). Additional support was provided by the SFB 840 .

\section{References}

1 K. R. Phillips, G. T. England, S. Sunny, E. Shirman, T. Shirman, N. Vogel and J. Aizenberg, Chem. Soc. Rev., 2016, 45, 281-322.

2 N. Vogel, M. Retsch, C. A. Fustin, A. Del Campo and U. Jonas, Chem. Rev., 2015, 115, 6265-6311.

3 Y. N. Xia, B. Gates, Y. D. Yin and Y. Lu, Adv. Mater., 2000, 12, 693-713.

4 J. F. Galisteo-López, M. Ibisate, R. Sapienza, L. S. FroufePérez, Á. Blanco and C. López, Adv. Mater., 2011, 23, 30-69.

5 A. Stein and R. C. Schroden, Curr. Opin. Solid State Mater. Sci., 2001, 5, 553-564.

6 S. Wong, V. Kitaev and G. A. Ozin, J. Am. Chem. Soc., 2003, 125, 15589-15598.

7 W. Cheng, J. Wang, U. Jonas, G. Fytas and N. Stefanou, Nat. Mater., 2006, 5, 830-836.

8 T. Still, W. Cheng, M. Retsch, R. Sainidou, J. Wang, U. Jonas, N. Stefanou and G. Fytas, Phys. Rev. Lett., 2008, 100, 194301.

9 K. Chen, T. Still, S. Schoenholz, K. B. Aptowicz, M. Schindler, A. C. Maggs, A. J. Liu and A. G. Yodh, Phys. Rev. E: Stat., Nonlinear, Soft Matter Phys., 2013, 88, 022315.

10 S.-M. Yang, S. G. Jang, D.-G. Choi, S. Kim and H. K. Yu, Small, 2006, 2, 458-475.

11 C. Stelling, A. Mark, G. Papastavrou and M. Retsch, Nanoscale, 2016, 8, 14556-14564.

12 M. Retsch and U. Jonas, Adv. Funct. Mater., 2013, 23, 5381-5389. 
13 F. A. Nutz, P. Ruckdeschel and M. Retsch, J. Colloid Interface Sci., 2015, 457, 96-101.

14 P. Ruckdeschel, T. W. Kemnitzer, F. A. Nutz, J. Senker and M. Retsch, Nanoscale, 2015, 7, 10059-10070.

15 P. A. Steward, J. Hearn and M. C. Wilkinson, Adv. Colloid Interface Sci., 2000, 86, 195-267.

16 M. A. Winnik, Curr. Opin. Colloid Interface Sci., 1997, 2, 192-199.

17 J. L. Keddie, Mater. Sci. Eng., R, 1997, 21, 101-170.

18 E. Gonzalez, M. Paulis, M. J. Barandiaran and J. L. Keddie, Langmuir, 2013, 29, 2044-2053.

19 R. E. Dillon, L. A. Matheson and E. B. Bradford, J. Colloid Sci., 1951, 6, 108-117.

20 P. Lepoutre and B. Alince, J. Appl. Polym. Sci., 1981, 26, 791-798.

21 X. Chen, S. Fischer, Z. Yi, V. Boyko, A. Terrenoire, F. Reinhold, J. Rieger, X. Li and Y. Men, Langmuir, 2011, 27, 8458-8463.

22 A. F. Routh and W. B. Russel, Langmuir, 1999, 15, 7762-7773.

23 A. F. Routh and W. B. Russel, Ind. Eng. Chem. Res., 2001, 40, 4302-4308.

24 D. P. Jensen and L. W. Morgan, J. Appl. Polym. Sci., 1991, 42, 2845-2849.

25 K. Hahn, G. Ley, H. Schuller and R. Oberthur, Colloid Polym. Sci., 1986, 264, 1092-1096.

26 J. E. Anderson and J. H. Jou, Macromolecules, 1987, 20, 1544-1549.

27 J. N. Yoo, L. H. Sperling, C. J. Glinka and A. Klein, Macromolecules, 1990, 23, 3962-3967.

28 K. D. Kim, L. H. Sperling, A. Klein and B. Hammouda, Macromolecules, 1994, 27, 6841-6850.

29 K. Hahn, G. Ley and R. Oberthur, Colloid Polym. Sci., 1988, 266, 631-639.

30 Q. Nawaz and Y. Rharbi, Langmuir, 2010, 26, 1226-1231.

31 Y. Rharbi, F. Boué and Q. Nawaz, Macromolecules, 2013, 46, $7812-7817$.
32 O. Pekcan, M. A. Winnik and M. D. Croucher, Macromolecules, 1990, 23, 2673-2678.

33 Y. C. Wang, C. L. Zhao and M. A. Winnik, J. Chem. Phys., 1991, 95, 2143-2153.

34 Y. C. Wang and M. A. Winnik, J. Phys. Chem., 1993, 97, 2507-2515.

35 E. M. Boczar, B. C. Dionne, Z. W. Fu, A. B. Kirk, P. M. Lesko and A. D. Koller, Macromolecules, 1993, 26, 5772-5781.

36 Y. S. Liu, J. R. Feng and M. A. Winnik, J. Chem. Phys., 1994, 101, 9096-9103.

37 W. L. Vos, M. Megens, C. M. van Kats and P. Bosecke, Langmuir, 1997, 13, 6004-6008.

38 N. Dingenouts and M. Ballauff, Langmuir, 1999, 15, 3283-3288.

39 S. Hu, J. Rieger, Z. Yi, J. Zhang, X. Chen, S. V. Roth, R. Gehrke and Y. Men, Langmuir, 2010, 26, 13216-13220.

40 E. A. Sulyanova, A. Shabalin, A. V. Zozulya, J. M. Meijer, D. Dzhigaev, O. Gorobtsov, R. P. Kurta, S. Lazarev, U. Lorenz, A. Singer, O. Yefanov, I. Zaluzhnyy, I. Besedin, M. Sprung, A. V. Petukhov and I. A. Vartanyants, Langmuir, 2015, 31, 5274-5283.

41 A. Georgiadis, P. A. Bryant, M. Murray, P. Beharrell and J. L. Keddie, Langmuir, 2011, 27, 2176-2180.

42 X. Qu, Y. Shi, Y. Tang, L. Chen and X. Jin, J. Colloid Interface Sci., 2002, 250, 484-491.

43 J. Rottstegge, B. Traub, M. Wilhelm, K. Landfester, C. Heldmann and H. W. Spiess, Macromol. Chem. Phys., 2003, 204, 787-802.

44 R. H. Ottewill and J. N. Shaw, Kolloid Z. Z. Polym., 1967, 215, 161-166.

45 T. Still, M. Retsch, U. Jonas, R. Sainidou, P. Rembert, K. Mpoukouvalas and G. Fytas, Macromolecules, 2010, 43, 3422-3428. 